capillary blood glucose was lowered significantly compared to the saline group, without alteration of infarct growth. In a secondary analysis, GKI was associated with significantly greater infarct growth in patients with complete intracranial vessel occlusion compared with controls ( $\mathrm{p}=0.011$ ) Brain lactate levels were significantly lower in GKI infused patients, and increased in control subjects. Asymptomatic hypoglycemia occurred in $76 \%$ of GKI-treated patients. (McCormick M, Hadley D, McLean JR, Macfarlane JA, Condon B, Muir KW. Randomized, controlled trial of insulin for acute poststroke hyperglycemia. Ann Neurol May 2010;67:570-578). (Respond: Prof Muir, Institute of Neurological Sciences, Southern General Hospital, Glasgow G51 4TF, Scotland, UK. Email: k.muir@clinmed.gla.ac.uk).

COMMENT. Hyperglycemia in more than $50 \%$ of acute stroke patients is an independent risk factor for poor outcome. Guidelines recommend blood glucose monitoring but optimal method of management is not established. In the above trial of insulin infusion within 24 hours of stroke, blood glucose was lowered and an increase in brain lactate was attenuated, but cerebral infarct growth was not affected. In patients with persistent arterial occlusion, GKI infusion was associated with greater infarct growth. The authors conclude that GKI infusion to treat moderate hyperglycemia in acute ischemic stroke requires further study and is not recommended in routine clinical practice.

An editorial (Johnston KC, Parsons M. Ann Neurol 2010;67:557-558) questions if the answer is in the imaging, and if intervention should be as early as possible after stroke onset. Studying the natural history of blood glucose after ischemic stroke, Wong AA et al (Neurology 2008;70:1036-1041) found that mean glucose levels remain static in patients with ischemic stroke without diabetes until at least 48 hours post-stroke. Levels are higher in patients with more severe stroke. Higher or lower levels regress to the mean over time.

\title{
DEGENERATIVE DISEASES
}

\section{EPIDEMIOLOGY OF PROGRESSIVE NEUROLOGICAL DISEASE}

Since 1997, researchers in the UK have searched for variant Creutzfeldt-Jacob and other diseases that cause progressive intellectual and neurological deterioration (PIND) by sending a monthly surveillance card to UK pediatricians. Clinical details are obtained by questionnaire or site visit. In 12 years, 2636 patients $<16$ years old with suspected PIND were reported, of whom 1114 had a confirmed diagnosis. Of 147 different diseases, the 6 commonest groups were leukoencephalopathies (183 cases), neuronal ceroid lipofuscinoses (141), mitochondrial (122), mucopolysaccharidoses (102), gangliosidoses (100), and perixisomal diseases (69). Districts having high rates of consanguinity reported relatively large numbers of PIND cases. Only 6 children with variant Creutzfeldt-Jacob disease were identified. (Verity C, Winstone AM, Stellitano L et al. The epidemiology of progressive intellectual and neurological deterioration in childhood. Arch Dis Child May 2010;95:361-364). (Respond: Dr CM Verity, Child Development Centre, Box 107, Addenbrooke's Hospital, Hills Road, Cambridge CB2 0QQ, UK. E-mail: christopher.verity@addenbrookes.nhs.uk). 